\title{
Optimization of graded multilayer designs for astronomical x-ray telescopes
}

\author{
Peter H. Mao, Fiona A. Harrison, David L. Windt, and Finn E. Christensen
}

\begin{abstract}
We developed a systematic method for optimizing the design of depth-graded multilayers for astronomical hard-x-ray and soft- $\gamma$-ray telescopes based on the instrument's bandpass and the field of view. We apply these methods to the design of the conical-approximation Wolter I optics employed by the balloon-borne High Energy Focusing Telescope, using W/Si as the multilayer materials. In addition, we present optimized performance calculations of mirrors, using other material pairs that are capable of extending performance to photon energies above the $\mathrm{W} K$-absorption edge $(69.5 \mathrm{keV})$, including $\mathrm{Pt} / \mathrm{C}, \mathrm{Ni} / \mathrm{C}, \mathrm{Cu} / \mathrm{Si}$, and Mo/Si. (C) 1999 Optical Society of America
\end{abstract}

OCIS codes: $\quad 110.6770,230.4170,340.7470,350.1260$.

\section{Introduction}

Present-generation astronomical hard-x-ray and soft$\gamma$-ray instruments employ either coded aperture masks or collimators to image x-ray sources. Because internal-detector background rates dominate typical source fluxes in this band, the faint source sensitivity is limited by the approximate equality of the collecting and the detector areas (1:1 for a collimator and 1:2 for a coded aperture). A focusing telescope, however, can have a collecting area that greatly exceeds the detector area (by factors of $10^{3}-$ $10^{4}$ ), thus resulting in significantly higher sensitivity. Several instruments are currently being developed to extend focusing capability into the hard-x-ray band. This includes at least two balloon instruments, InFocus, ${ }^{1}$ being developed by Goddard Space Flight Center and Nagoya University in Japan, and the High Energy Focusing Telescope (HEFT), ${ }^{2,3}$ being developed by Caltech, Columbia University, and the Danish Space Research Institute. In addition, the Constellation-X satellite mission ${ }^{4}$ is planning to incorporate a hard-x-ray focusing telescope.

The use of grazing incidence $\mathrm{x}$-ray telescopes at

P. H. Mao (peterm@srl.caltech.edu) and F. A. Harrison are with the Department of Physics, California Institute of Technology, MC 220-47, Pasadena, California 91125. D. L. Windt is with Bell Labs, Room 1D456, 600 Mountain Avenue, Murray Hill, New Jersey 07974. F. E. Christensen is with the Danish Space Research Institute, Juliane Maries Vej 30, Copenhagen DK-2100, Denmark.

Received 8 February 1999; revised manuscript received 12 May 1999.

0003-6935/99/224766-10\$15.00/0

(C) 1999 Optical Society of America high energy has been limited by the fact that the maximum incidence (or graze) angle for which significant reflectivity can be achieved with traditional metal coatings is roughly proportional to $1 / E$. Instruments relying on total external reflection to extend to significantly higher energy require small graze angles and therefore have a small ratio of mirror radius to focal length, $r / f$ [for a Wolter I or conical approximation mirror the maximum graze angle scales as $\left.\theta_{\max }=r /(4 f)\right]$. At some point it becomes impractical to achieve significant area for telescopes of reasonable focal length, and, in addition, the instrument field of view (proportional to $\theta_{\max }$ ) decreases.

Reflectivity at angles greater than the critical graze angle can, however, be achieved by use of depth-graded multilayer coatings on the mirror surfaces. ${ }^{5,6}$ Multilayer coatings consist of alternating layers of high- and low-refractive-index materials [e.g., tungsten and silicon (W/Si) or platinum and carbon $(\mathrm{Pt} / \mathrm{C})]$. As in Bragg reflection, the multilayer reflectivity results from constructive interference between reflections from adjacent layers. Achieving broad bandpass requires that the coating have a distribution of layer-pair (or bilayer) thicknesses in the direction of the normal vector to the surface. Because the number of interfaces required for a given level of reflectivity increases with the photon energy, the bilayer thicknesses in a graded multilayer are distributed with a greater number of thin layers. The distribution is arranged so that the higher-energy photons reflect at deeper layers in the stack, since higher-energy photons typically have lower absorption coefficients. Different approaches 
have been taken to determine the exact distribution of layer thicknesses, most notably, the power-law distribution by Joensen et al. ${ }^{7}$ and the sets of constant$d$-spacing blocks described by Yamashita et al. ${ }^{1}$

In this paper we describe a systematic method for optimizing the design of astronomical multilayer mirrors and describe its application to the balloon-borne HEFT conical-approximation Wolter I mirrors. In Section 2 we discuss the goals and constraints that guide the multilayer design. In Section 3 we describe our optimization technique, and in Section 4 we apply this technique to the HEFT optics and discuss the trade-offs incorporated into the design. Finally, in Section 5 we present alternatives to the current HEFT design that are capable of focusing to energies as high as $100 \mathrm{keV}$.

\section{General Design Considerations}

The optimum choice of multilayer materials and design of the bilayer thickness distribution depend critically on the desired mirror bandpass and field of view. Ultimately, however, the performance is limited by the physical properties of the materials, such as bulk densities, absorption cross sections, and chemical stability, as well as the technical limitations of multilayer fabrication: the minimum bilayer thickness for a given material pair and the deposition rates of the materials.

The range of bilayer thicknesses in a graded multilayer determines the bandpass and the field of view, and these are related by the Bragg equation. The refraction-corrected Bragg formula ${ }^{8}$ for a multilayer with constant bilayer thickness $d$, fractional thicknesses $\Gamma_{1}$ and $\Gamma_{2}$, and refractive indices $n_{1}=1-\delta_{1}-$ $i \beta_{1}$ and $n_{2}=1-\delta_{2}-i \beta_{2}$ is

$$
m \lambda=2 d \sin \theta\left[1-\frac{2\left(\Gamma_{1} \delta_{1}+\Gamma_{2} \delta_{2}\right)}{\sin ^{2} \theta}\right]^{1 / 2},
$$

where $m$ is the reflection order, $\lambda$ is the wavelength, and $\theta$ is the grazing incidence angle. By inverting the first-order $(m=1)$ Bragg equation for $d$, one can estimate the range in bilayer thicknesses required to reflect over the energy range $E_{\min }-E_{\text {max }}$ :

$$
\begin{aligned}
& d_{\min }=\frac{h c}{2 E_{\max } \sin \left(\theta_{\max }\right)}\left[1-\frac{2\left(\Gamma_{1} \delta_{1}+\Gamma_{2} \delta_{2}\right)}{\sin ^{2} \theta}\right]^{-1 / 2}, \\
& d_{\max }=\frac{h c}{2 E_{\min } \sin \left(\theta_{\min }\right)}\left[1-\frac{2\left(\Gamma_{1} \delta_{1}+\Gamma_{2} \delta_{2}\right)}{\sin ^{2} \theta}\right]^{-1 / 2} .
\end{aligned}
$$

In practice, because $\left[1-2\left(\Gamma_{1} \delta_{1}+\Gamma_{2} \delta_{2}\right) / \sin ^{2} \theta\right] \approx 1$, this term can be ignored when one determines the bilayer thickness range.

To use Eqs. (2) and (3) to calculate the range of bilayer thicknesses required for first-order reflection, one needs to know the range of incidence angles over which multilayer reflectivity (not total external reflection) is needed. Consider a single set of conicalapproximation Wolter I geometry mirrors with a primary mirror half-opening angle of $\alpha$ (see Fig. 1).

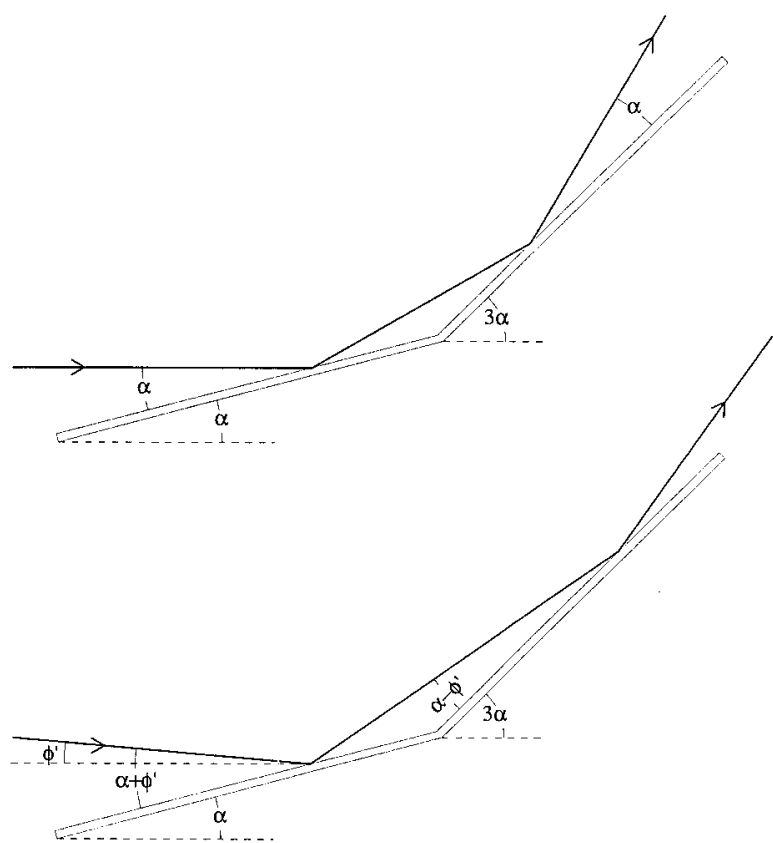

Fig. 1. On- and off-axis reflection geometry for a conical approximation to the Wolter I design. On-axis photons reflect at the same angle off the primary and the secondary mirrors. Off-axis photons that reflect at $\alpha+\phi^{\prime}$ off the primary mirror and whose directions of travel intersect the optical axis reflect at $\alpha-\phi^{\prime}$ off the secondary mirror. Off-axis photons that do not intersect the optical axis and reflect at $\alpha+\phi^{\prime}$ off the primary mirror reflect at angles within $2.5 \%$ of $\alpha-\phi^{\prime}$ off the secondary mirror.

For a field of view with half-angle $\phi$, the minimum and the maximum reflection angles are

$$
\begin{aligned}
\theta_{\min } & =\left\{\begin{array}{ll}
\alpha-\phi & \alpha-\phi>\theta_{\text {crit }} \\
\theta_{\text {crit }} & \alpha-\phi<\theta_{\text {crit }}
\end{array},\right. \\
\theta_{\max } & = \begin{cases}\alpha+\phi & \phi<\alpha \\
2 \alpha & \phi>\alpha\end{cases}
\end{aligned}
$$

respectively, where $\theta_{\text {crit }}$ is the critical angle for total external reflection. At angles less than $\theta_{\min }$ the photon is either out of the field of view $(\theta<\alpha-\phi)$ or in an angular range where it will reflect by total external reflection $\left(\theta<\theta_{\text {crit }}\right)$. At angles greater than $\theta_{\max }$ the photon is either out of the field of view $(\theta>\alpha+$ $\phi)$ or striking the primary mirror at an angle where it will not reflect off the secondary mirror and will not hit the focal spot $(\theta>2 \alpha)$.

For the maximum off-axis effective area, $d_{\text {min }}$ should be derived from Eq. (2), with $\theta_{\text {max }}$ given by Eq. (5). It can be difficult or impossible to fabricate the ideal minimum bilayer thicknesses. Performance is, however, not strongly degraded for minimum bilayer thicknesses that are somewhat larger than optimal. A small fraction of photons from a point source located at off-axis angle $\phi$ (assume, for simplicity, that $\phi<\alpha$ ) actually reflect off the mirror at an angle $\theta_{\text {max }}$. Furthermore, the distribution in the actual reflection angles from such a source is approximately uniform between the angles $\alpha-\phi$ and $\alpha+\phi$. The off-axis effective area is therefore 


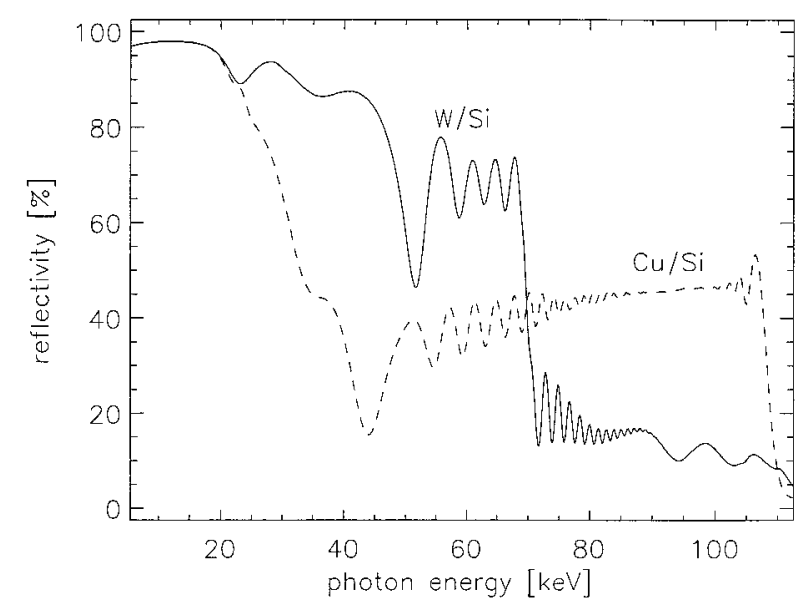

Fig. 2. Calculated reflectivity versus photon energy at $1.75 \mathrm{mrad}$ of a graded W/Si multilayer and a $\mathrm{Cu}-\mathrm{Si}$ multilayer with the exact same specifications (bilayer thickness distribution and interface width). The $\mathrm{Cu} / \mathrm{Si}$ reflectivity demonstrates that the range in bilayer thicknesses for this mirror would allow for reflectivity at $1.75 \mathrm{mrad}$ from 20 to $100 \mathrm{keV}$, but the jump in absorption at the $\mathrm{W} / K$ edge $(69.5 \mathrm{keV})$ drastically reduces reflectivity of the $\mathrm{W} / \mathrm{Si}$ multilayer above the absorption edge.

a weak function of the actual minimum bilayer thickness.

In choosing material combinations for a graded multilayer one must consider that the bandpass and the reflectivity are limited by attenuation in the multilayer, because of absorption and scattering at the interfaces. The ideal material pairs have a large difference in refractive index; minimal absorption over the energy range of interest; and can be fabricated with sharp, smooth interfaces. The first consideration, then, is to select material pairs with high mass density contrast and low absorption. The mass density ratio is a good indicator of the reflectivity per interface, since mass density is roughly proportional to electron density and since the reflectivity of an interface depends on the contrast in electron density across the interface. The absorption coefficient is important, because the reflectivity of a multilayer with highly absorbing materials will level off with fewer layer pairs. Thus, if the reflectivity per interface is the same, the material combination with a lower absorption coefficient will have better reflectance. Absorption is particularly acute when one of the multilayer materials has an atomic absorption edge in the energy range of interest. For example, at the W $K$ absorption edge $(69.5 \mathrm{keV})$, the reflectivity of a W/Si-graded multilayer drops considerably, as shown in Fig. 2. The reflectivity of a Cu/Si multilayer with the exact same specifications, shown as the dotted curve in Fig. 2, demonstrates that the cutoff is not due to the multilayer's bilayer distribution. A broadband reflector that uses tungsten is therefore limited either to energies below the $\mathrm{W}$ $K$ edge or significantly above it. An additional selection criterion is that the materials must produce multilayer films with minimal interdiffusion and interfacial roughness, imperfections that reduce reflectivity.

Finally, required coating thicknesses and deposition rates also factor into the multilayer design. In general the multilayer coating should be as thin as possible without sacrifice of performance. Thinner coatings and faster deposition rates allow for shorter coating times, both speeding up production and leaving the mirrors less exposed to variations in deposition conditions during coating.

\section{Optimization of Multilayer Designs}

To optimize the performance of depth-graded multilayers for astronomical hard-x-ray telescopes, we developed an optimization procedure that judges a design on the basis of its performance over the full energy range and field of view of the telescope. Previous discussions of multilayer design have not explicitly included the off-axis response of the mirrors in the design process.

\section{A. Figure of Merit Function}

To select optimum multilayer designs, we employ a figure of merit (FOM) based on the average reflectivity of the multilayer over the relevant energy range and field of view. We calculate the multilayer reflectivity with Fresnel coefficients, as described in Born and Wolf, ${ }^{9}$ using the physical constants tabulated at the Lawrence Livermore National Laboratory website. ${ }^{10}$ The formalism described in Born and Wolf assumes perfect interfaces; to include interdiffusion and roughness in the reflectivity calculations, we use the Névot-Croce model, ${ }^{11}$ where the reflectivity at an interface is reduced by a factor of $\exp \left[-(4 \pi \sigma / \lambda)^{2}\left(n_{i} \sin \theta_{i}\right)\left(n_{i+1} \sin \theta_{i+1}\right)\right]$. In the Névot-Croce, factor $n$ is the complex index of refraction; $i$ and $i+1$ are the layers above and below the interface, respectively; $\theta$ is the grazing incidence angle; and $\sigma$ is the interface width (which combines roughness and interdiffusion). Although in principle each interface width should be individually specified, in characterizing samples that we fabricated, we found that the data can be modeled by assuming a single value of $\sigma$ for all interfaces. A realistic value of $\sigma$ should be used for calculation of the reflectivity matrices, since this influences the outcome of the optimization procedure.

Given a matrix of reflectivities (a function of energy and angle), we calculate a single number, the FOM, which we use to compare designs. Our FOM is the integrated throughput of the mirrors over the energy band and field of view of the telescope. The primary complication in calculating the FOM is deciding on the weighting functions for performance versus both off-axis angle and energy. The choice of weighting functions depends intimately on the intended use of the telescope. For example, weighting the energy response by the atmospheric attenuation, which predominantly blocks low-energy x rays, is important for a balloon-borne telescope but not for a space-based telescope. In addition, because obtaining a given 
level of performance at low energies requires less coating thickness and far fewer layer pairs compared with the same performance at high energy, achieving a flat energy response requires skewing the weighting function to favor a high-energy response.

Clearly, the telescope geometry must be defined in order to determine how to weight the reflectivity versus off-axis angle. In this paper we consider applications to optics, using the conical approximation to the Wolter I geometry; however, in principle, this optimization technique is generally applicable to other systems. For the conical approximation to the Wolter I geometry, on-axis photons reflect off both sets of mirrors at the half-opening angle of the primary mirror $(\alpha)$. A photon that enters the optics with an off-axis angle of $\phi$ reflects off the primary mirror at an angle between $\alpha+\phi$ and $\alpha-\phi$, depending on where on the circumference it hits the mirror. If a photon reflects off the primary mirror with angle $\theta_{1}=\alpha+\phi^{\prime}$, then it reflects off the secondary mirror with angle $\theta_{2} \geq \alpha-\phi^{\prime}$. To simplify matters, we set $\theta_{2}=\alpha-\phi^{\prime}$, since in the worst-case situation for the HEFT design, i.e., large off-axis angle photons incident on the innermost mirror shell (4-cm radius, $\alpha=$ $1.67 \mathrm{mrad}$ ), the error in $\theta_{2}$ is less than $2.5 \%$. The geometry of these reflections is shown in Fig. 1. In addition, because of vignetting, we must determine for each mirror shell the incidence-angle distribution $\left(\theta_{1}, \theta_{2}\right)$ of photons that reach the detector as a function of off-axis angle $(\phi)$.

The FOM function we use to evaluate a design requires definition of a reflectivity matrix $[R(E, \theta)]$, a set of angular weighting functions $\left(W_{\alpha_{i} \phi}\right)$, and an energy-weighting function $\left(W_{E}\right)$. In addition, the energy response is further weighted by the atmospheric transmission function, $T_{\text {atm }}\left(E, \rho_{\text {atm }}\right)=$ $\exp \left[-\eta(E) \rho_{\text {atm }}\right]$, where $\eta(E)$ is the attenuation coefficient of dry air and $\rho_{\mathrm{atm}}$ is the altitude-dependent atmospheric column density. The FOM function, which incorporates all important design factors, is given by

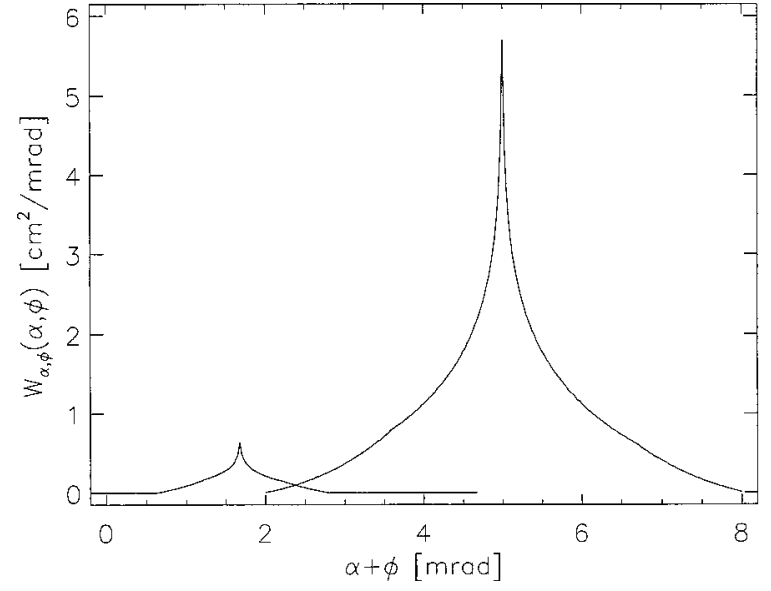

Fig. 3. Angular weighting function $\left(W_{\alpha_{i}, \phi}\right)$ for a uniform distribution of off-axis photons between 0 and $3 \mathrm{mrad}$ on the innermost $(\alpha=1.67 \mathrm{mrad})$ and the outermost $(\alpha=5.0 \mathrm{mrad})$ mirrors of the HEFT design.

scope aperture and recording of the primary mirror reflection angle of every photon that hits the focal plane within $0.5^{\prime}$ of the focal point. The distribution of off-axis angles used in the ray trace determines how off-axis response is weighted in the FOM. For example, a uniform distribution in off-axis photons evenly weights the FOM with respect to off-axis angles. By normalizing $W_{\alpha_{i}, \phi}$ so that $\sum_{\alpha_{i}} \int_{-\phi_{\max }}^{\phi_{\max }} \mathrm{d} \phi W_{\alpha_{i}, \phi}\left(\alpha_{i}, \phi\right)$ is the average effective area of the mirror shells over the field of view when $R=1$, we make the FOM proportional to the average effective area. Figure 3 shows the angular weighting functions, generated with a uniform distribution in off-axis angles between 0 and $3 \mathrm{mrad}$, of the innermost and the outermost mirror shells in HEFT.

In addition to the average throughput given by the FOM, it is also useful to calculate the energy dependence of the effective area, averaged over the field of view. The average effective area, $A\left(E, \rho_{\text {atm }}\right)$, is sim-

$$
\mathrm{FOM}=\frac{\sum_{\alpha_{i}} \int_{E_{\min }}^{E_{\max }} \mathrm{d} E \int_{-\phi_{\max }}^{\phi_{\max }} \mathrm{d} \phi T_{\mathrm{atm}}\left(E, \rho_{\mathrm{atm}}\right) R\left(E, \alpha_{i}+\phi\right) R\left(E, \alpha_{i}-\phi\right) W_{\alpha_{i}, \phi}\left(\alpha_{i}, \phi\right) W_{E}(E)}{\left\langle W_{E}\right\rangle\left(E_{\max }-E_{\min }\right)},
$$

where $\alpha_{i}$ is the half-opening angle of the $i$ th primary shell, $\phi_{\max }$ is the half-angle of the field of view, $\alpha_{i}+$ $\phi$ is the incidence angle on the $i$ th primary shell, and $\left\langle W_{E}\right\rangle$ denotes the average value of $W_{E}$ over the energy range $E_{\min }-E_{\max }$. The denominator in Eq. (6) normalizes the FOM to be independent of the normalization of $W_{E}$. $\quad W_{\alpha_{i}, \text { }}$ is the incidence-angle distribution on the $i$ th shell over the field of view and incorporates the geometry of the optics, including the thicknesses of the substrates and their spacing. $W_{\alpha_{i}, \phi}$ is generated by uniform illumination of the tele- ply the FOM with the energy integral, $W_{E}$, and the denominator omitted:

$$
\begin{aligned}
A\left(E, \rho_{\text {atm }}\right)= & T_{\text {atm }}\left(E, \rho_{\text {atm }}\right) \sum_{\alpha_{i}} \int_{-\phi_{\max }}^{\phi_{\max }} \mathrm{d} \phi W_{\alpha_{i}, \phi}\left(\alpha_{i}, \phi\right) \\
& \times R\left(E, \alpha_{i}+\phi\right) R\left(E, \alpha_{i}-\phi\right) .
\end{aligned}
$$

We use $A\left(E, \rho_{\text {atm }}\right)$ to compare optimum designs with near-optimum designs and to compare the relative performance of different material combinations. 


\section{B. Multilayer Parameterization}

For a selected material pair, energy range, and field of view, globally optimizing the multilayer design (given $W_{E}$ and $W_{\alpha, \phi}$ ) is a daunting task. The phase space of possible designs is extraordinarily large, $2 N$ variables for a multilayer with $N$ bilayers, and the number of layer pairs for optimal performance is not known a priori. In addition, the computational complexity of the problem scales with $N$. The problem can be made more tractable by parameterization of the layer thickness distribution.

Graded multilayers were originally used as neutron reflectors, and one method for parameterizing the layer thickness distribution, developed by Joensen et al., ${ }^{7}$ which we adopt here, is a generalization of Mezei's parameterization for flat, broadband response neutron mirrors. ${ }^{12}$ In Joensen's parameterization the bilayer thickness distribution is defined by a power law with three parameters,

$$
d(i)=a /(b+i)^{c},
$$

where $a, b$, and $c$ are the constants and $i$ is the bilayer index ranging from 1 to $N$, with $i=N$ being the bilayer next to the substrate. A complete description of a (perfect-interface) multilayer requires, in addition, specification of the fractional thicknesses within each bilayer. To simplify matters, we set the high- $Z$-thickness fraction $(\Gamma)$ of each bilayer to a constant. Equation (8) defines the bilayer thickness distribution with the definition of four of six possible parameters: $a, b, c, N, d_{\min }$, or $d_{\max }$. It is convenient to use $c, N, d_{\min }$, and $d_{\max }$ to specify the bilayer distribution. If we fix $c, d_{\min }, d_{\max }$, and $\Gamma$, then by varying $N$ we can compare similar bilayer distributions. These sets of distributions are similar in the sense that they share a common continuous distribution and $N$ is the number of times the continuous distribution is sampled. Joensen's parameterization thus reduces the number of parameters needed to define a graded multilayer from $2 N$ to 5 .

Another method for designing multilayers, used by Yamashita et al., ${ }^{1}$ is to define a series of constant$d$-spacing blocks. In Yamashita's method the five thickest layers, at the top of the stack, are graded, and there are three sets of layers with constant bilayer thickness within each set. This method results in a multilayer design with an approximately power-law distribution in the bilayers. For our designs we chose to use Joensen's method, because it allows for a more systematic approach to optimizing the layer thickness distribution.

\section{Optimization Technique}

The computational time and resources required for a full optimization, even with the problem simplified by Joensen's parameterization, are substantial: The reflectivity matrix for an $N=250$ multilayer takes 6 min on a $333-\mathrm{MHz}$ Sun Enterprise 3000 server, a comprehensive optimization requires searching over five parameters $\left(N, c, d_{\min }, d_{\max }, \Gamma\right)$, and the time to calculate a reflectivity matrix for one set of parame- ters is proportional to $N$. Also, one would ideally design a different multilayer for each of the 60-100 nested mirror shells in a Wolter I telescope, since each shell's opening angle is different. We reduce the number of different designs to 10 by designing for small groups of shells instead of for individual shells. We further reduce the parameter space by setting the bilayer thickness range for each group of shells ahead of time and optimizing on only three parameters: the number of bilayers $(N)$, the power-law index $(c)$ from Eq. (8), and the high- $Z$-thickness fraction $(\Gamma)$.

Ideally, Eqs. (2) and (3) alone would set the bilayer thickness range. In practice, however, technical limitations such as the minimum achievable bilayer thickness and the desire for short deposition times dictate setting $d_{\min }$ to a value greater than that given by Eqs. (2) and (5). Using a larger value of $d_{\text {min }}$ invariably reduces the optimum FOM, since the mirror will no longer reflect high-energy photons at large off-axis angles. As discussed in Section 2, however, the loss in performance is usually acceptable, because, for any given off-axis angle, reflections at the largest angles only account for a small fraction of the photons reaching the detector. Furthermore, because the bilayer thickness distribution has a powerlaw form, a small change in $d_{\text {min }}$ results in a drastic reduction in coating thickness.

With the mirror groups established and values of $d_{\text {min }}$ and $d_{\max }$ chosen for each group, finding the optimum distribution is simply a matter of calculating reflectivity matrices and FOM's for several values of $N, c$, and $\Gamma$. With only three parameters to optimize, we calculate reflectivity matrices in $(N, c, \Gamma)$ space on a grid at intervals of 50 in $N, 0.005$ in $c$, and 0.05 in $\Gamma$. Our optimization program uses the Message Passing Interface Library ${ }^{13}$ to calculate FOM at points in $(N, c, \Gamma)$ space in parallel. We originally developed and ran this code on our local network, using 1-10 processors at a time, before porting the program to the parallel computers at Caltech's Center for Advanced Computing Research (CACR). Our code returns values of FOM over a large region of $(N$, $c, \Gamma$ ) space, allowing us to identify the global optimum design coordinates in the $d_{\min }-d_{\max }$ constrained space and giving us the information necessary to make quantifiable trade-offs between performance and coating time, as we show in Section 4.

Our current approach does not make the best use of processor time, since the calculation time is proportional to the largest value of $N$ that we specify. In the future we will change the code to calculate the reflectivity matrices in parallel. This makes better use of processor time and will lead to an efficient iterative optimization in which the time to calculate each step is proportional to $N /$ (number of processors).

\section{High Energy Focusing Telescope Design}

We illustrate the optimization process by describing its application to HEFT. HEFT is a balloon-borne hard-x-ray telescope with a 6-m focal length and consisting of 14 identical coaligned telescope modules. In each module there are 71 mirror shells between 4 
Table 1. HEFT Design: Mirror Shell Groups and Bilayer Thickness Ranges

\begin{tabular}{|c|c|c|c|c|c|c|c|}
\hline $\begin{array}{l}\text { Mirror } \\
\text { Group }\end{array}$ & $\begin{array}{c}\text { Angular Range } \\
\text { (mrad) }\end{array}$ & $\begin{array}{l}\text { Radial Range } \\
(\mathrm{cm})\end{array}$ & $\begin{array}{l}\text { Shells per } \\
\text { Module }\end{array}$ & $\begin{array}{c}d_{\min }(\AA) \\
\left(\text { with } \theta_{\max }=\alpha_{\max }\right)\end{array}$ & $\begin{array}{c}d_{\min }(\AA) \\
\text { (adjusted) }\end{array}$ & $\Delta d_{\min }$ & $d_{\max }$ \\
\hline 1 & $1.67-1.86$ & $4.00-4.46$ & 6 & 47.6 & 33.3 & $-30 \%$ & 297.6 \\
\hline 2 & $1.86-2.08$ & $4.46-4.99$ & 6 & 42.7 & 29.9 & $-30 \%$ & 266.6 \\
\hline 3 & $2.08-2.32$ & $4.99-5.57$ & 7 & 38.2 & 28.7 & $-25 \%$ & 238.9 \\
\hline 4 & $2.32-2.59$ & $5.57-6.22$ & 7 & 34.2 & 27.4 & $-20 \%$ & 214.0 \\
\hline 5 & $2.59-2.89$ & $6.22-6.94$ & 7 & 30.7 & 26.1 & $-15 \%$ & 191.8 \\
\hline 6 & $2.89-3.22$ & $6.94-7.73$ & 7 & 27.5 & 24.7 & $-10 \%$ & 171.8 \\
\hline 7 & $3.22-3.60$ & $7.73-8.64$ & 7 & 24.6 & 24.6 & $0 \%$ & 153.9 \\
\hline 8 & $3.60-4.01$ & $8.64-9.62$ & 8 & 22.1 & 24.3 & $+10 \%$ & 137.9 \\
\hline 9 & $4.01-4.48$ & $9.62-10.75$ & 8 & 19.8 & 23.7 & $+20 \%$ & 123.6 \\
\hline 10 & $4.48-5.00$ & $10.75-12.0$ & 8 & 17.7 & 23.0 & $+30 \%$ & 110.7 \\
\hline
\end{tabular}

and $12 \mathrm{~cm}$ in radius (half-opening angles of 1.67-5.0 mrad) configured in a conical-approximation Wolter I geometry. The HEFT science goals include broadband spectroscopy and imaging of extended sources. HEFT therefore requires multilayer designs optimized for broadband reflectivity, from the atmospheric cutoff (near $20 \mathrm{keV}$ at 130,000 ft) to as high an energy as possible, over a wide range of incidence angles. For the multilayer this means covering the widest possible range in bilayer thicknesses. Because of the small achievable interface widths $(\sigma=$ 3.0-3.5 ̊) and because both components have high deposition rates $(\geq 1 \AA / \mathrm{s})$, we selected W/Si for HEFT. This choice does, however, impose a cutoff in the effective area at the W $K$ edge $(69.5 \mathrm{keV})$. Before we run the optimization code, we must define the angular and the energy weighting functions and determine appropriate values of $d_{\min }$ and $d_{\max }$ for each group of mirror shells. Our procedure gives us the optimum and the near-optimum designs under the given constraints, which include a coating thickness target of 1.0-1.5 $\mu \mathrm{m}$ per coating imposed by the HEFT production schedule. Throughout our W/Si calculations we use $\sigma=3.5 \AA$, a conservative choice for this material combination.

\section{A. Weighting Functions}

With perfect reflectivity the geometry of the HEFT optics results in a field of view where the effective area at $3 \mathrm{mrad}$ off axis is half of the on-axis effective area. Accordingly, we generate $W_{\alpha_{i}, \phi}$ by ray tracing with a uniform 0-3 mrad (off-axis angle) input photon distribution. This is the same angular weighting function described in Subsection 3.A and shown in Fig. 3 for the innermost and the outermost mirror shells. If the mission were solely dedicated to detecting extended sources, we would have chosen a distribution with a weighting function that increases with off-axis angle.

For the energy-weighting function we use $W_{E}=$ $E[\mathrm{keV}]+70$. As we discussed in Subsection 3.A, a low-energy response is easier to obtain than a highenergy response, so for a balanced broadband design we use a $W_{E}$ that favors a high-energy response to some extent. Our choice of the linear function is, however, somewhat arbitrary, and one may find that, for other applications, steeper or shallower functions will be appropriate.

The atmospheric transmission factor $\left(T_{\mathrm{atm}}\right)$ is required for the FOM to represent accurately the actual throughput of a balloon-borne telescope. At low energies $(\sim 20 \mathrm{keV})$ reflectivity may be close to $100 \%$, but the spectrum is severely attenuated; if $T_{\text {atm }}=1$, then changes in the low-energy response would greatly affect the FOM but would have almost no bearing on the true performance of the telescope. We use $T_{\text {atm }}$ with $\rho_{\text {atm }}=3.5 \mathrm{~g} / \mathrm{cm}^{2}$, the atmospheric column density at an altitude of $130,000 \mathrm{ft}$. For a satellite mission, $\rho_{\text {atm }} \approx 0 \mathrm{~g} / \mathrm{cm}^{2}$, making $T_{\text {atm }}$ unity.

\section{B. Shell Groupings and Bilayer Thickness Ranges}

We divided the HEFT mirror shells into ten groups (a reasonable compromise between the complexity of the calculation and optimal performance), logarithmically spaced in opening angles. The angular and the radial ranges of each group of shells, along with the number of shells (per module) in each group, are listed in Table 1.

We specify the maximum bilayer thickness $\left(d_{\max }\right)$ for each group of shells to the value given by Eq. (3) with $E_{\min }=25 \mathrm{keV}$ and $\theta_{\min }=\alpha_{\min } / 2$, where $\alpha_{\min }$ is the half-opening angle of the smallest radius shell in the group. The $\theta_{\min }$ we use is larger than the value given by Eq. (4), but we found that specifying $d_{\max }$ in this way gives the mirror response a smooth transition between small angles (or energies) where reflectivity is due to total external reflection and to larger angles (or energies) where reflectivity depends on the multilayer design. With smaller values of $d_{\max }$ the on-axis reflectivity of the mirror shells exhibits a strong dip near $E_{\text {min }}$.

Calculating $d_{\min }$ with Eqs. (2) and (5) results in optimized designs with impractically thick coatings. As discussed in Section 2 and Subsection 3.C, specifying a larger value of $d_{\min }$ reduces both the optimized FOM and the coating thickness. Although it would be best to optimize $d_{\text {min }}$ explicitly by employment of a coating thickness constraint, this would significantly increase computation time; therefore we make educated guesses to determine $d_{\min }$, as outlined below.

As a starting point we use Eq. (2) with $E_{\max }=70$ 

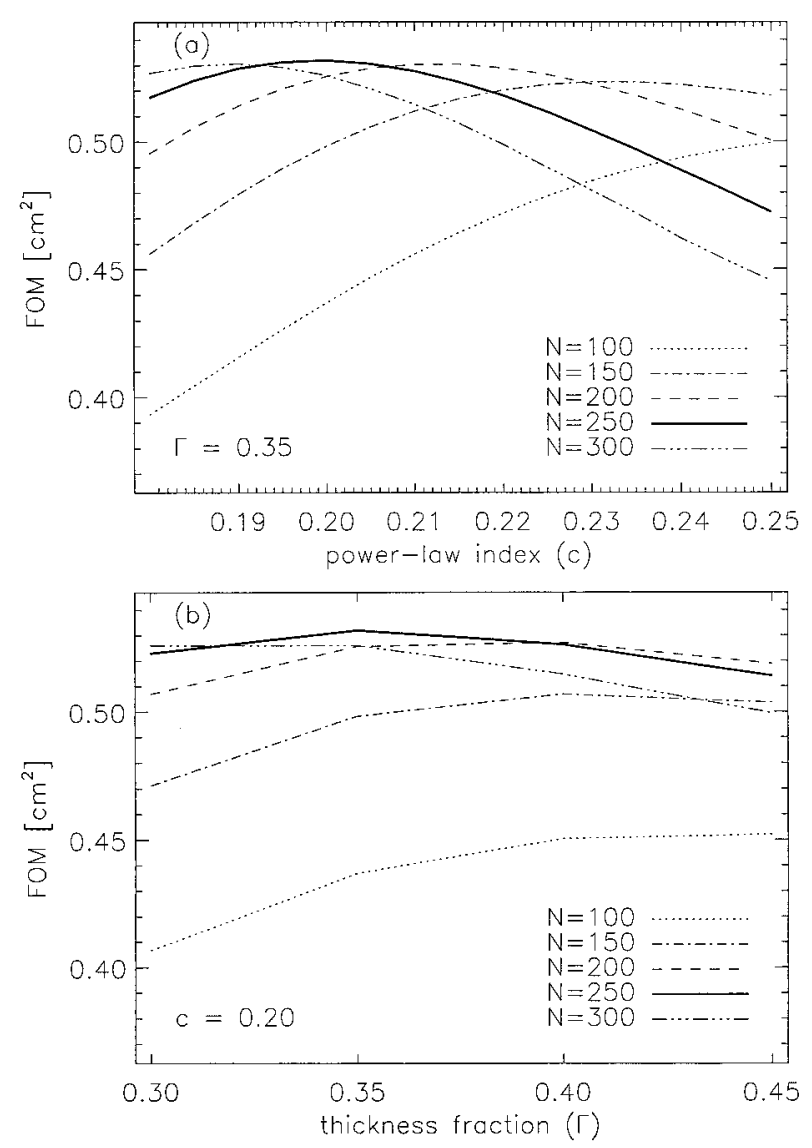

Fig. 4. Mirror group 1 FOM calculations for $\mathrm{W} / \mathrm{Si}$ with $d_{\min }=$ $33.3 \AA, d_{\max }=297.6 \AA$, and $\sigma=3.5 \AA$. The FOM is calculated over the energy range $20-70 \mathrm{keV}$ with $\rho_{\text {atm }}=3.5 \mathrm{~g} / \mathrm{cm}^{2}$. Under these conditions the optimal design is $1.06 \mu \mathrm{m}$ thick with $N=250$, $c=0.20$, and $\Gamma=0.35$. (a) FOM versus $c$ for $N=150$ to $N=300$ with $\Gamma=0.35$. (b) FOM versus $\Gamma$ for $N=150$ to $N=300$ with $c=$ 0.20 .

$\mathrm{keV}$ and $\theta_{\max }=\alpha_{\max }$, where $\alpha_{\max }$ is the half-opening angle of the largest radius shell in the group. The resulting values of $d_{\text {min }}$ allow for first-order Bragg reflection over the entire energy band for on-axis sources, but they result in reduced off-axis response at high energies. Optimized designs are thinner on the inner groups of shells and thicker on the outer ones, so we bracket the range in coating thicknesses for this set of specifications by finding the optimum design parameters $(N, c$, and $\Gamma)$ for groups 1 and 10 . With the $\theta_{\max }=\alpha_{\max }$ criteria the optimized design for group $1(N=50, c=0.185, \Gamma=0.35)$ is $0.31 \mu \mathrm{m}$ thick, with a FOM of 0.498 , and the optimum group 10 design $(N=1850, c=0.175, \Gamma=0.35)$ is $3.97 \mu \mathrm{m}$ thick, with a FOM of 0.721 . This indicates that we can substantially reduce $d_{\min }$ on the inner shells, thus improving the FOM. On the outer shells we must reduce the coating thickness by either raising $d_{\text {min }}$ or dropping $N$. We choose to trade performance for coating thickness on the outer mirror groups by raising $d_{\text {min }}$. The adjustments to $d_{\min }$ from our initial choices, which bring the optimum thicknesses closer to $1 \mu \mathrm{m}$, are listed in Table 1 .

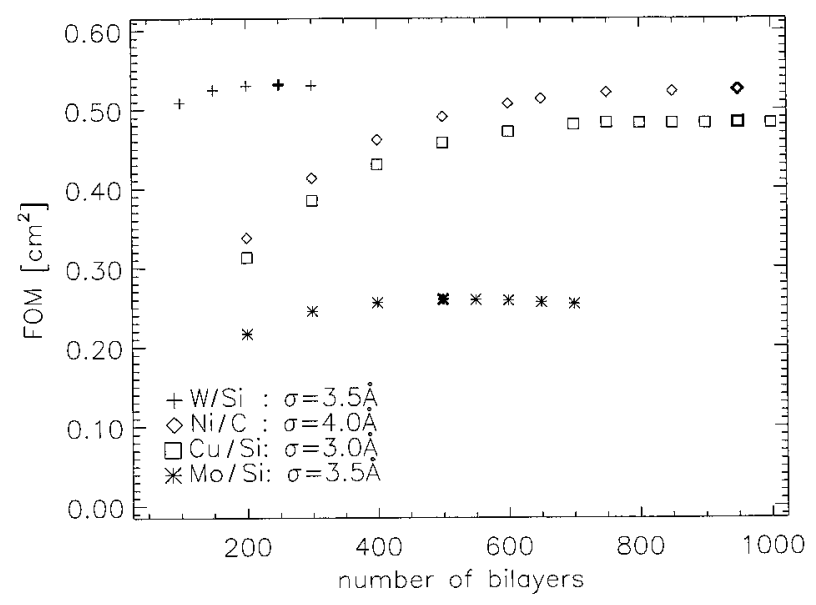

Fig. 5. $\operatorname{FOM}(N)$ versus $N$ for mirror group 1 designs with $d_{\min }=$ $33.3 \AA$ and $d_{\text {max }}=297.6 \AA$. The weighting functions are as described in the text. $\mathrm{W} / \mathrm{Si}$ is optimized over the energy range 20-70 keV. The other materials are optimized over the energy range $20-100 \mathrm{keV}$. Each marker indicates the optimum FOM (allowing for $c$ and $\Gamma$ to vary) for a multilayer with the given number of bilayers. The bold markers indicate the optimum designs.

We stress that our choices of $d_{\min }$ and $d_{\text {max }}$ do not result in globally optimal designs - the coatings of such designs would be far too thick. Practical considerations force us to sacrifice performance, and under these constraints we search for values of the remaining free parameters $(N, c$, and $\Gamma)$ that optimize the overall FOM for the coatings. The advantage of our systematic approach is that we can quantify the performance difference between the theoretically optimal designs and designs compromised by practical constraints and therefore make educated decisions in trading performance against schedule.

\section{Optimization Results and Discussion}

With the weighting functions, mirror shell groups, and bilayer thickness ranges determined, we calculate the FOM for several points in $(N, c, \Gamma)$ space as described in Subsection 3.C. For a given set of constraints the FOM does not increase monotonically with $N$ indefinitely, but rather there is an optimal number of layers beyond which absorption degrades the performance. As an example of the optimization output we show two calculations of the mirror group 1 performance in Figs. 4(a) and 4(b): The first shows FOM versus $c$ with $\Gamma=0.35$ for several values of $N$, and the second shows FOM versus $\Gamma$ with $c=0.20$ for several values of $N$. These calculations also demonstrate that the FOM is generally a smooth function of $N$, $c$, and $\Gamma$, without local maxima that would lead to incorrect results with iterative optimization routines.

It is useful to analyze how the FOM varies with coating thickness for a given set of constraints. Coating thickness is roughly proportional to $N$; it is independent of $\Gamma$ and a weak function of $c$. By plotting $\operatorname{FOM}(N)$ versus $N$, where $\operatorname{FOM}(N)$ is the maximum FOM for a given value of $N$, we can make informed trade-offs between the performance of a de- 


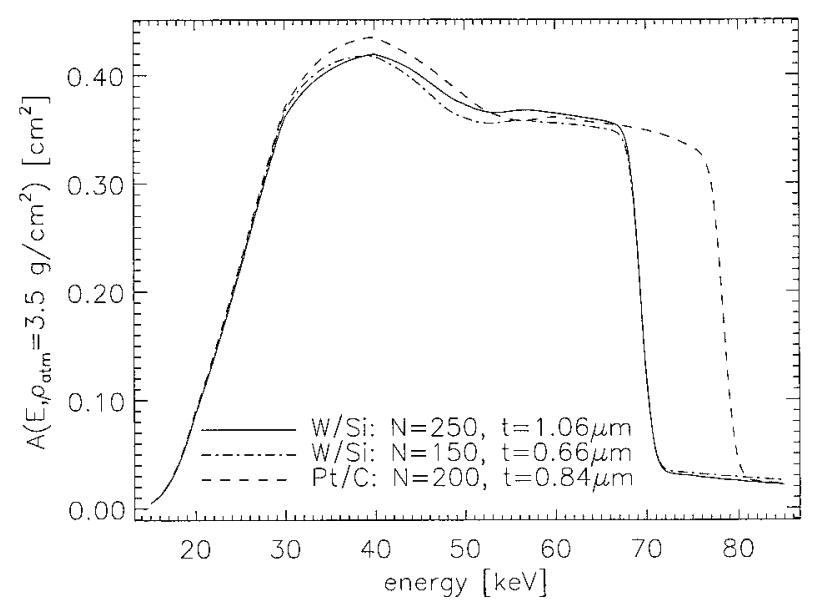

Fig. 6. Average effective area versus energy calculations of the optimum (solid curve) and near-optimum (dotted-dashed curve) $\mathrm{W} /$ Si multilayer designs for mirror group 1 . For parameters see Table 2. Also shown is the performance of the optimum $\mathrm{Pt} / \mathrm{C}$ multilayer (dashed curve) with the same bilayer thickness range but optimized for $20-80 \mathrm{keV}$. The parameters of the $\mathrm{Pt} / \mathrm{C}$ design are $c=0.20$ and $\Gamma=0.30$.

sign and its thickness. The $\operatorname{FOM}(N)$ versus $N$ plot for mirror group 1 (pluses in Fig. 5) indicates that the optimum design has 250 bilayers but that the best 150 bilayer design has a FOM that is $99 \%$ of the optimum design. The average effective areas $[A(E$, $\left.\left.\rho_{\text {atm }}=3.5 \mathrm{~g} / \mathrm{cm}^{2}\right)\right]$ for mirror group 1's optimum and near-optimum designs, plotted in Fig. 6, demonstrate that the performance of these two designs is nearly identical. Clearly, if coating time is an issue, the 150 bilayer design, which is $38 \%$ thinner than the optimum 250 bilayer design, would be preferred.

The parameters for two possible HEFT mirror designs are listed in Table 2. The design on the lefthand side is the optimal parameter set (given the constraints), and the one on the right-hand side is a reduced coating thickness near-optimal design. The optimal design has an average coating thickness of $1.34 \mu \mathrm{m}$, whereas the average thickness of the nearoptimal design, which has comparable performance (see Fig. 7), is only $0.97 \mu \mathrm{m}$.

\section{Designs for Extending to Higher Energies}

The choice of $\mathrm{W} / \mathrm{Si}$ limits the HEFT design to energies below the $\mathrm{W} K$ edge. Several scientific objectives require effective areas extending to $100 \mathrm{keV}$ or even beyond. This requires either finding a suitable material pair (without a $K$-absorption edge in the energy range of interest) or developing other $\mathrm{W} / \mathrm{Si}$ multilayer distributions that achieve a high-energy response at the expense of a low-energy response.

Alternatives to $\mathrm{W} / \mathrm{Si}$ include $\mathrm{Cu} / \mathrm{Si},{ }^{14,15} \mathrm{Mo} / \mathrm{Si}$, $\mathrm{Ni} / \mathrm{C}$, and $\mathrm{Pt} / \mathrm{C}$. With the exception of $\mathrm{Pt} / \mathrm{C}$, which is limited by the Pt $K$ edge $(78.4 \mathrm{keV})$, these materials are all capable of broadband reflectivity from $20 \mathrm{keV}$ to $100 \mathrm{keV}$ and above. Table 3 lists the density ratios and the absorption coefficients at $30 \mathrm{keV}^{16}$ for the material combinations that we investigated. On the basis of these numbers one can quickly determine which material combinations behave similarly (those with comparable density ratios and absorption coefficients, i.e., W/Si versus $\mathrm{Pt} / \mathrm{C}$ and $\mathrm{Cu} / \mathrm{Si}$ versus $\mathrm{Ni} / \mathrm{C})$ and which combinations are unfavorable (those with absorption coefficients that are high relative to their density ratio, i.e., $\mathrm{Mo} / \mathrm{Si}$ ).

Figure 6 compares the performance of W/Si (solid curve) with $\mathrm{Pt} / \mathrm{C}$ (dotted curve) for the innermost shells (group 1) of the HEFT design. The average throughput $\left[A\left(E, \rho_{\mathrm{atm}}=3.5 \mathrm{~g} / \mathrm{cm}^{2}\right)\right]$ shown for these material pairs is calculated from the optimum designs derived from the same weighting functions used in the HEFT design. $\mathrm{Pt} / \mathrm{C}$ interface widths between 3.0 and $5.0 \AA$ were reported by Yamashita et al. ${ }^{17}$; in these calculations we use $\sigma=3.5 \AA$. As we expect from density ratios and absorption coefficients, under the same conditions, the optimum designs for these two material pairs have nearly the same coating

Table 2. HEFT Design Parameters for W-Si

\begin{tabular}{|c|c|c|c|c|c|c|c|c|c|c|}
\hline \multirow{2}{*}{$\begin{array}{l}\text { Mirror } \\
\text { Group }\end{array}$} & \multicolumn{5}{|c|}{ Optimum Design $^{a}$} & \multicolumn{5}{|c|}{ Near-Optimum Design ${ }^{b}$} \\
\hline & $N^{c}$ & $c^{d}$ & $\Gamma^{e}$ & $t^{f}$ & FOM & $N$ & $c$ & $\Gamma$ & $t$ & FOM \\
\hline 1 & 250 & 0.200 & 0.35 & 1.06 & 0.532 & 150 & 0.225 & 0.40 & 0.66 & 0.525 \\
\hline 2 & 350 & 0.205 & 0.35 & 1.33 & 0.609 & 200 & 0.230 & 0.40 & 0.79 & 0.600 \\
\hline 3 & 350 & 0.210 & 0.35 & 1.28 & 0.796 & 250 & 0.220 & 0.40 & 0.93 & 0.790 \\
\hline 4 & 400 & 0.205 & 0.35 & 1.39 & 0.865 & 250 & 0.225 & 0.40 & 0.90 & 0.852 \\
\hline 5 & 450 & 0.200 & 0.35 & 1.48 & 0.905 & 300 & 0.220 & 0.40 & 1.01 & 0.893 \\
\hline 6 & 500 & 0.195 & 0.35 & 1.54 & 0.913 & 350 & 0.215 & 0.40 & 1.11 & 0.899 \\
\hline 7 & 450 & 0.195 & 0.35 & 1.38 & 0.865 & 350 & 0.200 & 0.40 & 1.08 & 0.852 \\
\hline 8 & 450 & 0.190 & 0.35 & 1.36 & 0.867 & 350 & 0.205 & 0.35 & 1.08 & 0.858 \\
\hline 9 & 450 & 0.185 & 0.35 & 1.31 & 0.725 & 350 & 0.200 & 0.35 & 1.04 & 0.710 \\
\hline 10 & 450 & 0.180 & 0.35 & 1.27 & 0.588 & 350 & 0.195 & 0.35 & 1.00 & 0.574 \\
\hline
\end{tabular}

${ }^{a}$ With adjusted $d_{\text {min }}$ from Table 1 .

${ }^{b}$ Parameters resulting in FOM that are within $98 \%$ of the optimal design's FOM.

${ }^{c}$ Number of bilayers.

${ }^{d}$ Power-law index [see Eq. (8)].

${ }^{e}$ High-Z- (heavy-element) layer thickness fraction.

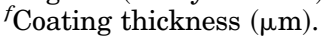




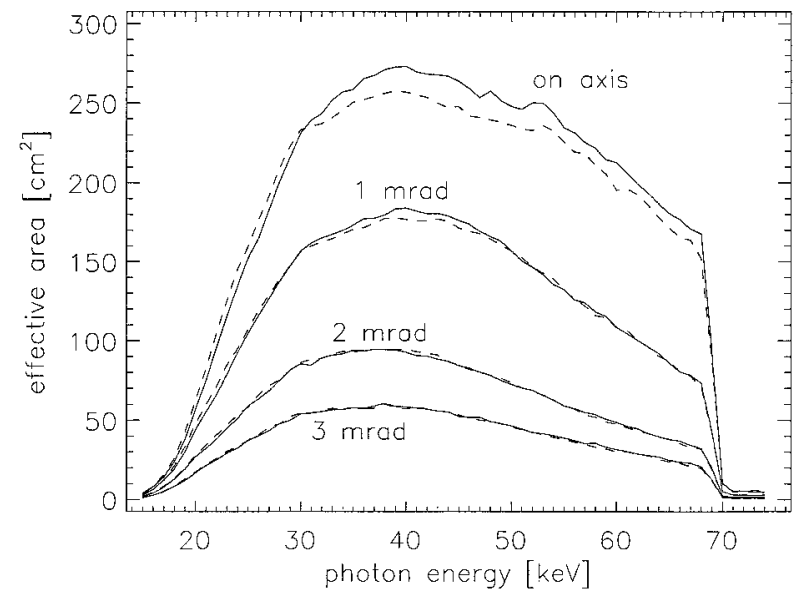

Fig. 7. Calculated effective area of the optimum (solid curves) and near-optimum (dashed curves) HEFT multilayer mirror designs for an on-axis point source and for point sources at 1.0, 2.0, and 3.0 mrad off axis.

thicknesses: $1.06 \mu \mathrm{m}$ for $\mathrm{W} / \mathrm{Si}$ and $0.84 \mu \mathrm{m}$ for $\mathrm{Pt} / \mathrm{C}$. Also, as we expect, the material pairs give comparable performance up to the W $K$ edge $(69.5$ $\mathrm{keV}$ ), where absorption in the $\mathrm{W} / \mathrm{Si}$ multilayer cuts off reflectivity at higher energies. The $\mathrm{Pt} / \mathrm{C}$ response is cut off at $78.4 \mathrm{keV}$ by the $\mathrm{Pt} K$-absorption edge.

We also ran optimizations on $\mathrm{Mo} / \mathrm{Si}, \mathrm{Cu} / \mathrm{Si}$, and $\mathrm{Ni} / \mathrm{C}$ for group 1 mirrors. The multilayers use the same bilayer thickness ranges as in the HEFT design but are optimized over the $20-100-\mathrm{keV}$ energy band. The interface widths used in the reflectivity calculations represent our best results with these materials to date $[\sigma(\mathrm{Ni} / \mathrm{C})=4.0 \AA, \sigma(\mathrm{Mo} / \mathrm{Si})=3.5 \AA, \sigma(\mathrm{Cu} / \mathrm{Si})$ $=3.0 \AA]$. $\quad \operatorname{FOM}(N)$ versus $N$ for these sets of materials is plotted in Fig. 5 with the corresponding values of $c$ and $\Gamma$ omitted for clarity. $\mathrm{Cu} / \mathrm{Si}$ and $\mathrm{Ni} / \mathrm{C}$ optimize at much greater coating thicknesses than Mo/Si because of their lower absorption coefficients $(t=4.05 \mu \mathrm{m}$ with $N=950, c=0.215$, and $\Gamma=0.35$ for $\mathrm{Cu} / \mathrm{Si}$ and Ni/C; $t=2.11 \mu \mathrm{m}$ with $N=500, c=$ 0.205 , and $\Gamma=0.35$ for $\mathrm{Mo} / \mathrm{Si}$ ). Even so, if we compare designs with approximately the same coating thicknesses, $\mathrm{Cu} / \mathrm{Si}$ and $\mathrm{Ni} / \mathrm{C}$ are still substantially better reflectors than $\mathrm{Mo} / \mathrm{Si}$. The average throughput of the best $N=500 \mathrm{Ni} / \mathrm{C}, \mathrm{Cu} / \mathrm{Si}$, and $\mathrm{Mo} / \mathrm{Si}$

Table 3. Comparison of the Physical Properties of a Few Multilayer Material Combinations ${ }^{a}$

\begin{tabular}{cccc}
\hline Materials & $\rho_{1} / \rho_{2}$ & $\begin{array}{c}\mu_{1} \\
\left(\mathrm{~cm}^{-1}\right)^{b}\end{array}$ & $\begin{array}{c}\mu_{2} \\
\left(\mathrm{~cm}^{-1}\right)^{c}\end{array}$ \\
\hline $\mathrm{Pt} / \mathrm{C}$ & 9.77 & 566 & 0.435 \\
$\mathrm{~W} / \mathrm{Si}$ & 8.28 & 439 & 3.35 \\
$\mathrm{Mo} / \mathrm{Si}$ & 4.38 & 287 & 3.35 \\
$\mathrm{Ni} / \mathrm{C}$ & 4.05 & 92.0 & 0.435 \\
$\mathrm{Cu} / \mathrm{Si}$ & 3.85 & 97.8 & 3.35 \\
\hline
\end{tabular}

${ }^{a}$ Ref. 16 .

${ }^{b}$ Absorption coefficient of the high-Z material.

${ }^{c}$ Absorption coefficient of the low-Z material.

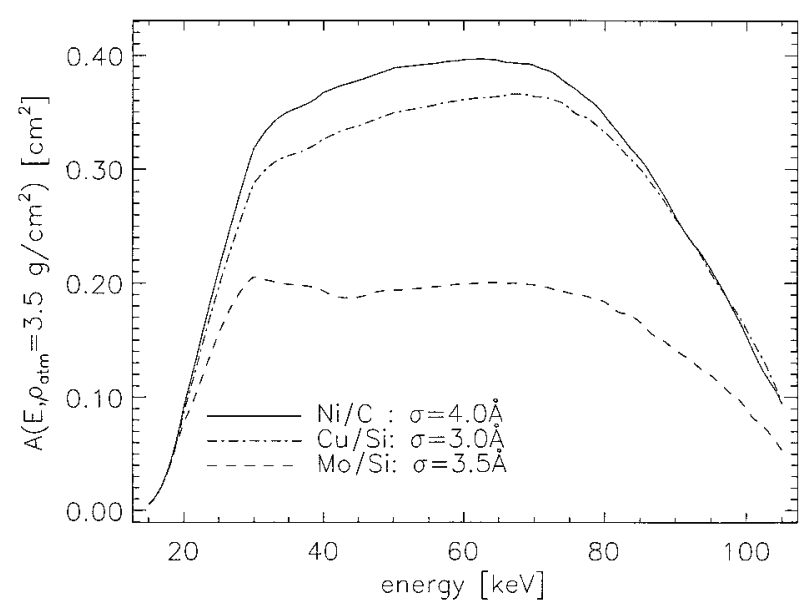

Fig. 8. Comparison of $A\left(E, \rho_{\text {atm }}=3.5 \mathrm{~g} / \mathrm{cm}^{2}\right)$ of the best $N=500$ $\mathrm{Ni} / \mathrm{C}, \mathrm{Cu} / \mathrm{Si}$, and $\mathrm{Mo} / \mathrm{Si}$ multilayers designed for mirror group 1. The coating thicknesses of these designs are all between 2.10 and $2.25 \mu \mathrm{m}$.

designs are plotted in Fig. 8. The results reflect what we expect, given the values in Table $3: \mathrm{Ni} / \mathrm{C}$ $(c=0.25, \Gamma=0.45)$ gives the best performance, closely followed by $\mathrm{Cu} / \mathrm{Si}(c=0.24, \Gamma=0.45)$, and the relatively poor performance of $\mathrm{Mo} / \mathrm{Si}$ is obviously due to the high-Mo-absorption cross section. If coating time were not an issue, $\mathrm{Ni} / \mathrm{C}$ would be the optimal choice for 20-100-keV designs; however, $\mathrm{Cu} / \mathrm{Si}$ can be coated much more quickly, and, given the comparable performance with $\mathrm{Ni} / \mathrm{C}$, is preferable on practical grounds.

Using a W/Si multilayer designed to reflect at 70 $100 \mathrm{keV}$ is another possibility for extending the effective area beyond the W $K$ edge; however, it requires additional trade-offs, and the advantage of this approach is therefore unclear. Although we have not investigated such designs in great detail, a high-energy W/Si multilayer would have an on-axis collecting area comparable with that of $\mathrm{Ni} / \mathrm{C}$ or $\mathrm{Cu} / \mathrm{Si}$ in the $70-100-\mathrm{keV}$ range but with a much thinner coating. The disadvantage is that one sacrifices the entire 20-70-keV range. A possible remedy for the loss in low-energy response would be to use a $20-70$ $\mathrm{keV} \mathrm{W/Si-graded} \mathrm{coating} \mathrm{underneath} \mathrm{the} \mathrm{70-100-}$ $\mathrm{keV}$ coating; however, this would increase the coating thickness substantially, thus negating the advantage of a thinner coating.

\section{Conclusion}

We have derived a formula [Eq. (6)] for calculating the average effective area of a conical-approximation Wolter I telescope over its field of view and bandpass. Using this formula, we have developed a systematic method of searching for optimal-graded multilayer designs that does not neglect off-axis performance. The phase space of possible designs is formidably large, requiring specification of the number of bilayers and $2 N$ individual layer thicknesses. We simplify the problem by using Joensen's power-law parameterization of bilayer thicknesses [Eq. (8)]. 
We apply our optimization method to the design of HEFT mirrors, using W/Si. The final design incorporates trade-offs between overall performance and total coating thickness. Employing the figure of merit (FOM) function allows us to quantify trade-offs between performance and coating thickness. We have also investigated the performance of several alternatives to $\mathrm{W} / \mathrm{Si}$. $\mathrm{Pt} / \mathrm{C}$ and $\mathrm{Ni} / \mathrm{C}$ both give excellent performance, but the higher deposition rate of silicon relative to carbon makes $\mathrm{Cu} / \mathrm{Si}$ appear to be the best choice for mirrors that reflect at energies above the W $K$ edge. Mo/Si, although popular for softer-x-ray mirrors, performs poorly above $20 \mathrm{keV}$, because of its high absorption coefficient.

This research was supported in part by NASA Supporting Research and Technology grant NAG5-5128 and by a NASA Graduate Student Researchers Program Fellowship (to Peter H. Mao). This research was performed in part with the CACR parallel computer system operated by Caltech. Peter H. Mao thanks Thomas Prince for access to CACR's services and Stuart Anderson for advice and guidance on parallel computing codes. We also thank Steve Boggs and the anonymous referees for helpful comments on the manuscript.

\section{References}

1. K. Yamashita, P. J. Serlemitsos, J. Tueller, S. D. Barthelmy, L. M. Bartlett, K. W. Chan, A. Furuzawa, N. Gehrels, K. Haga, H. Kunieda, P. Kurczynski, G. Lodha, N. Nakajo, N. Nakamura, Y. Namba, Y. Ogasaka, T. Okajima, D. Palmer, A. Parsons, Y. Soong, S. M. Stahl, H. Takata, K. Tamura, Y. Tawara, and B. J. Teegarden, "Supermirror hard-x-ray telescope," Appl. Opt. 37, 8067-8073 (1998).

2. P. H. Mao, F. A. Harrison, Y. Y. Platonov, D. Broadway, B. Degroot, F. E. Christensen, W. W. Craig, and C. J. Hailey, "Development of grazing incidence multilayer mirrors for hard X-ray focusing telescopes," in EUV, X-Ray, and Gamma-Ray Instrumentation for Astronomy VIII, O. H. Siegmund and M. A. Gummin, eds., Proc. SPIE 3114, 526-534 (1997).

3. C. J. Hailey, S. Abdali, F. E. Christensen, W. W. Craig, T. R. Decker, F. A. Harrison, and M. Jimenez-Garate, "Substrates and mounting techniques for the High-Energy Focusing Telescope (HEFT)," in EUV, X-Ray, and Gamma-Ray Instrumentation for Astronomy VIII, O. H. Siegmund and M. A. Gummin, eds., Proc. SPIE 3114, 535-543 (1997).
4. H. Tananbaum, N. White, and P. Sullivan, eds., Proceedings of the High Throughput X-ray Spectroscopy Workshop (HarvardSmithsonian Center for Astrophysics, Cambridge, Mass, 1996).

5. F. E. Christensen, A. Hornstrup, N. J. Westergaard, H. W. Schnopper, J. L. Wood, and K. Parker, "A graded d-spacing multilayer telescope for high energy X-ray astronomy," in Multilayer and Grazing Incidence X-ray/EUV Optics, R. B. Hoover, ed., Proc. SPIE 1546, 160-167 (1992).

6. K. D. Joensen, F. E. Christensen, H. W. Schnopper, P. Gorenstein, J. Susini, P. Hoghoj, R. Hustache, J. L. Wood, and K. Parker, "Medium-sized grazing incidence high-energy X-ray telescopes employing continuously graded multilayers," in $X$-Ray Detector Physics and Applications, R. B. Hoover, ed., Proc. SPIE 1736, 239-248 (1993).

7. K. D. Joensen, P. Voutov, A. Szentgyorgyi, J. Roll, P. Gorenstein, P. Hoghoj, and F. E. Christensen, "Design of grazingincidence multilayer supermirrors for hard-X-ray reflectors," Appl. Opt. 34, 7935-7944 (1995).

8. E. Spiller, Soft X-ray Optics (Society of Photo-Optical Instrumentation Engineers, Bellingham, Wash., 1994).

9. M. Born and E. Wolf, Principles of Optics, 6th ed. (Pergamon, New York, 1993).

10. L. Kissel and P. M. Bergstrom, Jr., "Anomalous scattering factors," http://www-phys.llnl.gov/V_Div/scattering/asf.html.

11. L. Névot and P. Croce, "Charactérisation des surfaces par refléxion rasante de rayons $\mathrm{X}$. Application á l'étude du polissage de quelques verres silicates," Rev. Phys. Appl. 15, 761779 (1980).

12. F. Mezei, "Novel polarized neutron devices: supermirror and spin component amplifier," Comment Phys. 1, 81-85 (1976).

13. W. Gropp, E. Lusk, and A. Skjellum, Using MPI: Portable Parallel Programming with the Message-Passing Interface (MIT Press, Cambridge, Mass., 1994).

14. D. L. Windt, "Multilayer X-ray optics," in Crystal and Multilayer Optics, A. T. Macrander, D. M. Mills, A. K. Freund, and T. Ishikawa, eds., Proc. SPIE 3448, 280-290 (1998).

15. D. L. Windt, "Cu/Si depth-graded multilayers for hard X-ray mirrors," Appl. Phys. Lett. 74, 2890-2892 (1999).

16. J. H. Hubbell and S. M. Seltzer, "Tables of X-ray mass attenuation coefficients and mass energy-absorption coefficients," Tech. Rep. NISTIR 5632, web version 1.02, National Institute of Standards and Technology http://physics.nist.gov/PhysRefData/XrayMassCoef/cover.html.

17. K. Yamashita, K. Akiyama, K. Haga, H. Kunieda, G. S. Lodha, N. Nakajo, N. Nakamura, T. Okajima, K. Tamura, and Y. Tawara, "Fabrication and characterization of multilayer supermirrors for hard X-ray optics," J. Synchrotron Rad. 5, 711713 (1998). 